\title{
EQUI-ATTRACTION AND THE CONTINUOUS DEPENDENCE OF ATTRACTORS ON PARAMETERS
}

\author{
LI DESHENG* \\ Department of Mathematics and Information Science, Yantai University, Yantai 264005, \\ Shandong, People's Republic of China \\ e-mail:lids@ytu.edu.cn \\ and P. E. KLOEDEN \\ FB Mathematik, Johann Wolfgang Goethe Universität, D-60054 Frankfurt am Main, Germany \\ e-mail:kloeden@math.uni-frankfurt.de
}

(Received 16 October, 2002; accepted 2 February, 2003)

\begin{abstract}
The equi-attraction properties concerning the global attractors $\mathcal{A}_{\lambda}$ of dynamical systems $S_{\lambda}(t)$ with parameter $\lambda \in \Lambda$, where $\Lambda$ is a compact metric space, are investigated. In particular, under appropriate conditions, it is shown that the equiattraction of the family $\left\{\mathcal{A}_{\lambda}\right\}$ is equivalent to the continuity of $\mathcal{A}_{\lambda}$ in $\lambda$ with respect to the Hausdorff distance.
\end{abstract}

2000 Mathematics Subject Classification. Primary 47J25, 37L30, 34C20.

1. Introduction. Let $\lambda$ be a parameter belonging to a compact metric space $\Lambda$ with metric $\rho$ and let $\mathbb{T}=\mathbb{R}^{+}$or $\mathbb{Z}^{+}$. Let $\left\{S_{\lambda}(t)\right\}(t \in \mathbb{T})$ be a family of dynamical systems (semigroups) on a complete metric space $X$ with metric $d$, where the parameter $\lambda \in \Lambda$. For any $x \in X$ and $A \subset X$, set $d(x, A)=\inf _{y \in A} d(x, y)$. Denote by $d_{H}$ and $\delta_{H}$ the Hausdorff semidistance and Hausdorff distance on $X$, which are defined, respectively, by

$$
d_{H}\left(A_{1}, A_{2}\right)=\sup _{x \in A_{1}} d\left(x, A_{2}\right), \quad \delta_{H}\left(A_{1}, A_{2}\right)=\max \left\{d_{H}\left(A_{1}, A_{2}\right), d_{H}\left(A_{2}, A_{1}\right)\right\}
$$

for any $A_{1}, A_{2} \subset X$

Suppose that $S_{\lambda}(t)$ has a global attractor $\mathcal{A}_{\lambda}$. Then, for each $\lambda \in \Lambda, \mathcal{A}_{\lambda}$ attracts an arbitrary bounded subset $B$ of $X$ under $S_{\lambda}(t)$; that is, for every $\varepsilon>0$, there exists a $\tau=\tau_{\lambda}(B, \varepsilon)>0$ such that

$$
d_{H}\left(S_{\lambda}(t) B, \mathcal{A}_{\lambda}\right)<\varepsilon, \quad \forall t \geq \tau .
$$

Let $B$ be a bounded subset of $X$. If for any $\varepsilon>0$, there exists a $\tau=\tau(B, \varepsilon)>0$ independent of $\lambda \in \Lambda$ such that (1.1) holds for all $\lambda \in \Lambda$, then we say that $\mathcal{A}_{\lambda}$ equiattracts $B$ for $\lambda \in \Lambda$, or, the family $\left\{\mathcal{A}_{\lambda}\right\}$ equi-attracts $B$. In case $\left\{\mathcal{A}_{\lambda}\right\}$ equi-attracts each bounded subset $B$ of $X$, we simply say that $\left\{\mathcal{A}_{\lambda}\right\}$ is equi-attracting.

If the family of global attractors of dynamical systems $S_{\lambda}(t)$ with parameter $\lambda$ is equi-attracting, then a singularity does not appear in the rate of attraction as $\lambda$ varies.

*Supported by the National Natural Science Foundation of China (10251002). 
This is clearly important in the approximation of dynamical behavior, e.g., $[\mathbf{4}, \mathbf{5}, \mathbf{1 0}$, 13, 20]. However, it is generally not true and the time $\tau$ appearing in (1.1) may depend sensitively on $\lambda$. This can be seen even in some simple situations such as Example 3.2 below.

In the present paper, instead of considering the attractors $\mathcal{A}_{\lambda}$ themselves, we investigate the following parameterically inflated attractors, namely, the sets

$$
\mathcal{A}_{\lambda}[r]:=\bigcup_{\rho\left(\lambda^{\prime}, \lambda\right) \leq r} \mathcal{A}_{\lambda^{\prime}}
$$

for $r \geq 0$. Assume the family of dynamical systems $\left\{S_{\lambda}(t)\right\}(\lambda \in \Lambda)$ is equi-dissipative on $X$ and uniformly compact for $t$ large (see Section 2 for formal definitions). Then, under some additional continuity assumptions, we will prove that for any fixed $r>0,\left\{\mathcal{A}_{\lambda}[r]\right\}$ equi-attracts each bounded subset $B$ of $X$, i.e., for any $\varepsilon>0$, there is a $\tau=\tau(B, \varepsilon)>0$ independent of $\lambda$ such that

$$
d_{H}\left(S_{\lambda}(t) B, \mathcal{A}_{\lambda}[r]\right)<\varepsilon, \quad \forall t \geq \tau
$$

for all $\lambda \in \Lambda$. Some of the ideas used here come from an earlier work [16] of the first co-author.

In case $r=0$, we have by the definition of $\mathcal{A}_{\lambda}[r]$ that $\mathcal{A}_{\lambda}[0]=\mathcal{A}_{\lambda}$. As it has been pointed out, the family $\left\{\mathcal{A}_{\lambda}\right\}$ may not possess the equi-attraction property. However, based on the above result, we will show that if $\mathcal{A}_{\lambda}$ is continuous in $\lambda$, then $\left\{\mathcal{A}_{\lambda}\right\}$ is equi-attracting. As a direct application of this basic fact, we will further prove under some reasonable continuity assumptions that the family $\left\{\mathcal{A}_{\lambda}\right\}$ is uniformly Lyapunov stable. This indicates that the Lyapunov stability of $\mathcal{A}_{\lambda}$ is robust with respect to the variation of $\lambda$ in $\Lambda$. Uniform Lyapunov stability of attractors for perturbed systems aroused notable interest in recent years; see, for instance, Kostin and Pilyugin [14].

The continuity of attractors under perturbation or discretization is a crucial issue in the theory of dynamical systems; see $[3,7,8,9,10,12,15]$ and [17] etc. As a consequence of our results, we finally prove that under appropriate conditions, the continuity of the global attractor $\mathcal{A}_{\lambda}$ in $\lambda$ with respect to the Hausdorff distance is equivalent to the equi-attraction property of the family $\left\{\mathcal{A}_{\lambda}\right\}$. This can be regarded as an inherent characterization of the continuous dependence of attractors on a parameter. Similar considerations on the attractors $\mathcal{A}_{\lambda}$ for discretized dynamical systems with constant stepsize $\lambda \geq 0$ can be found in the works of Grüne $[\mathbf{4}, \mathbf{5}]$ and also in Stuart and Humphries [20] etc., where the authors provide conditions ensuring continuity of $\mathcal{A}_{\lambda}$ as $\lambda$ goes to 0 , which are formulated in terms of attraction rate of these attractors.

Part of the main results here have already been used successfully to investigate the uniform dynamics of periodic and asymptotically periodic non-autonomous systems. This will be reported in a forthcoming paper [23].

This paper is organized as follows. In Section 2 we state and prove the main results, and then in Section 3 we illustrate the results in Section 2 in terms of involving parametrized ordinary differential equations.

2. The Main Results. In this section we state and prove our main results. We first present some definitions. 
Definition 2.1. Let $S(t)(t \in \mathbb{T})$ be a dynamical system (semigroup) on $X$. A compact subset $\mathcal{A}$ is called a global attractor of $S(t)$, if $S(t) \mathcal{A}=\mathcal{A}$ for all $t \in \mathbb{T}$, and $\mathcal{A}$ attracts each bounded subset $B$ of $X$.

We refer the reader to $[\mathbf{2}, \mathbf{6}, \mathbf{7}, \mathbf{1 8}, \mathbf{1 9}, \mathbf{2 1}]$ and $[\mathbf{2 2}]$ etc. for the basic theory of global attractors. Note that the global attractor of a system, if exists, is unique.

Definition 2.2. Let $\left\{S_{\lambda}(t)\right\}(\lambda \in \Lambda)$ be a family of dynamical systems on $X$. We say that

(1) $\left\{S_{\lambda}(t)\right\}$ is equi-dissipative on $X$ if there exists a bounded subset $\mathcal{U}$ of $X$ so that for any bounded subset $B \subset X$, there exists a $t_{0}=t_{0}(B) \in \mathbb{T}$ independent of $\lambda \in \Lambda$ such that

$$
S_{\lambda}(t) B \subset \mathcal{U}, \quad t \geq t_{0}
$$

(2) $\left\{S_{\lambda}(t)\right\}$ is uniformly compact for $t$ large if for any bounded subset $B$ of $X$, there exists a $t_{0}=t_{0}(B) \in \mathbb{T}$ independent of $\lambda \in \Lambda$, such that $\bigcup_{\lambda \in \Lambda} S_{\lambda}(t) B$ is relatively compact for any $t \geq t_{0}$.

Our first theorem concerns the paramaterically inflated attractors $\mathcal{A}_{\lambda}[r]$ in case $r>0$.

THEOREM 2.3. Assume that $\left\{S_{\lambda}(t)\right\}(\lambda \in \Lambda)$ is equi-dissipative and uniformly compact for t large. In addition, assume that

(C1) For any $t \in \mathbb{T}$ fixed, $S_{\lambda}(t) x$ is jointly continuous in $(x, \lambda)$ on $X \times \Lambda$.

Let $\mathcal{A}_{\lambda}$ be the global attractor of $S_{\lambda}(t)$. Then for any $r>0$ fixed, the family $\left\{\mathcal{A}_{\lambda}[r]\right\}$ is equi-attracting.

Proof. Let $r>0$. We need to prove that for any bounded subset $B$ of $X$ and $\varepsilon>0$, there exists a $\tau=\tau(B, \varepsilon)$ which is independent of $\lambda \in \Lambda$ such that

$$
d_{H}\left(S_{\lambda}(t) B, \mathcal{A}_{\lambda}[r]\right)<\varepsilon, \quad \forall t \geq \tau, \lambda \in \Lambda
$$

For convenience, we assume that $\varepsilon<r$. Set $M=X \times \Lambda$ and endow $M$ with the metric $\sigma$ defined by

$$
\sigma\left((x, \lambda),\left(y, \lambda^{\prime}\right)\right)=d(x, y)+\rho\left(\lambda, \lambda^{\prime}\right), \quad \forall(x, \lambda),\left(y, \lambda^{\prime}\right) \in M .
$$

Clearly $(M, \sigma)$ is complete. Consider the semigroup $G(t)(t \in \mathbb{T})$ on $M$,

$$
G(t)(x, \lambda)=\left(S_{\lambda}(t) x, \lambda\right), \quad \forall(x, \lambda) \in M .
$$

$G(t)$ is well defined on $M$ and due to the joint continuity of $S_{\lambda}(t) x$ in $(x, \lambda)$, we see that for any fixed $t \in \mathbb{T}, G(t)$ is a continuous mapping on $M$. Since $\left\{S_{\lambda}(t)\right\}$ is equi-dissipative, there exists a bounded subset $\mathcal{U}$ of $X$ such that for any bounded subset $A$ of $X$, one can find a $t_{0}=t_{0}(A) \in \mathbb{T}$ in dependent of $\lambda$ so that

$$
S_{\lambda}(t) A \subset \mathcal{U}, \quad \forall t \geq t_{0}, \lambda \in \Lambda .
$$

It follows that $\Omega:=\mathcal{U} \times \Lambda$ is a bounded absorbing set of $G(t)$, i.e., for each bounded subset $K$ of $M, G(t) K \subset \Omega$ for $t$ sufficiently large. The uniform compactness of $\left\{S_{\lambda}(t)\right\}$ implies that $G(t)$ is compact when $t$ is sufficiently large (recall that $\Lambda$ is compact). 
In particular, there exists a $t_{1} \in \mathbb{T}$ such that $G\left(t_{1}\right) \Omega$ is relatively compact. Let $K$ be a bounded subset of $M$. Take a $t_{2} \in \mathbb{T}$ such that $G(t) K \subset \Omega$ for $t \geq t_{2}$. Observing that

$$
\bigcup_{t \geq t_{1}+t_{2}} G(t) K=G\left(t_{1}\right)\left(\cup_{t \geq t_{2}} G(t) K\right) \subset G\left(t_{1}\right) \Omega,
$$

we find that $\bigcup_{t \geq t_{1}+t_{2}} G(t) K$ is relatively compact. Thus by basic results in the theory of dynamical systems (see $[\mathbf{7}, \mathbf{1 1}, \mathbf{2 1}]$ etc.), $G(t)$ has a unique global attractor $\Theta$.

Let

$$
\mathcal{A}_{\lambda}^{\prime}=\{x \in X:(x, \lambda) \in \Theta\} .
$$

As $\Theta$ is invariant under $G(t)$, i.e., $G(t) \Theta=\Theta$ for any $t \in \mathbb{T}$, by the definition of $G(t)$, we find that $\mathcal{A}_{\lambda}^{\prime}$ is invariant under $S_{\lambda}(t)$; and thus $\mathcal{A}_{\lambda}^{\prime} \subset \mathcal{A}_{\lambda}$. On the other hand, by the invariance of $\mathcal{A}_{\lambda}$ under $S_{\lambda}(t)$ one can easily see that $\mathcal{A}_{\lambda} \times\{\lambda\}$ is invariant under $G(t)$. Therefore $\mathcal{A}_{\lambda} \times\{\lambda\} \subset \Theta$. It follows that $\mathcal{A}_{\lambda} \subset \mathcal{A}_{\lambda}^{\prime}$. Hence $\mathcal{A}_{\lambda}^{\prime}=\mathcal{A}_{\lambda}$. In conclusion, we have

$$
\Theta=\bigcup_{\lambda \in \Lambda} \mathcal{A}_{\lambda} \times\{\lambda\}
$$

As $\Theta$ attracts $\Omega$ under $G(t)$, there exists $\tau_{0}=\tau_{0}(\Omega, \varepsilon) \in \mathbb{T}$ such that when $t \geq \tau_{0}$, we have

$$
\inf _{v \in \Theta} \sigma(G(t) u, v)<\varepsilon / 2, \quad \forall u \in \Omega .
$$

For any $\lambda \in \Lambda$, we divide $\Theta$ into two parts:

$$
\Theta=\left(\bigcup_{\rho\left(\lambda^{\prime}, \lambda\right) \leq r} \mathcal{A}_{\lambda^{\prime}} \times\left\{\lambda^{\prime}\right\}\right) \cup\left(\bigcup_{\rho\left(\lambda^{\prime}, \lambda\right)>r} \mathcal{A}_{\lambda^{\prime}} \times\left\{\lambda^{\prime}\right\}\right):=\Theta_{\lambda}[r] \cup \Theta_{\lambda}^{c}[r] .
$$

Then (2.3) is to say that for any $t \geq \tau_{0}$,

$$
\inf _{v \in \Theta_{\lambda}[r] \cup \Theta_{\lambda}^{c}[r]} \sigma\left(\left(S_{\lambda}(t) x, \lambda\right), v\right)<\varepsilon / 2, \quad \forall(x, \lambda) \in \mathcal{U} \times \Lambda .
$$

Now suppose that $(x, \lambda) \in \mathcal{U} \times \Lambda$ and $t \geq \tau_{0}$. Noting that if $v:=\left(y, \lambda^{\prime}\right) \in \Theta_{\lambda}^{c}[r]$, then by the definition of $\sigma$,

$$
\sigma\left(\left(S_{\lambda}(t) x, \lambda\right), v\right) \geq \rho\left(\lambda, \lambda^{\prime}\right)>r>\varepsilon,
$$

by (2.4) we necessarily have

$$
\inf _{v \in \Theta_{\lambda}[r]} \sigma\left(\left(S_{\lambda}(t) x, \lambda\right), v\right)<\varepsilon / 2 .
$$

By using (2.6), one can find a $v:=\left(y, \lambda^{\prime}\right) \in \Theta_{\lambda}[r]$ such that

$$
\sigma\left(\left(S_{\lambda}(t) x, \lambda\right),\left(y, \lambda^{\prime}\right)\right)<2 \varepsilon / 3 \text {. }
$$

Noticing that $\rho\left(\lambda^{\prime}, \lambda\right) \leq r$ and hence $y \in \mathcal{A}_{\lambda^{\prime}} \subset \mathcal{A}_{\lambda}[r]$, we deduce by (2.7) that

$$
d_{H}\left(S_{\lambda}(t) x, \mathcal{A}_{\lambda}[r]\right) \leq d\left(S_{\lambda}(t) x, y\right) \leq \sigma\left(\left(S_{\lambda}(t) x, \lambda\right),\left(y, \lambda^{\prime}\right)\right)<2 \varepsilon / 3 .
$$


Since $x, \lambda$ and $t$ are arbitrary, we conclude that

$$
d_{H}\left(S_{\lambda}(t) \mathcal{U}, \mathcal{A}_{\lambda}[r]\right) \leq 2 \varepsilon / 3<\varepsilon, \quad \forall t \geq \tau_{0}, \lambda \in \Lambda .
$$

This and (2.2) finish the proof of (2.1). The proof of the theorem is complete.

Now we consider the case $r=0$ in which $\mathcal{A}_{\lambda}[0]=\mathcal{A}_{\lambda}$. As pointed out in the introduction, $\left\{\mathcal{A}_{\lambda}\right\}$ may not possess equi-attraction property in general. However, if $\mathcal{A}_{\lambda}$ is continuous in $\lambda$ in the sense of Hausdorff distance, then we can show that it is equi-attracting.

THEOREM 2.4. Assume the hypotheses in Theorem 2.3. Let $\mathcal{A}_{\lambda}$ be the global attractor of $S_{\lambda}(t)$. If $\mathcal{A}_{\lambda}$ is continuous in $\lambda$ with respect to the Hausdorff distance, then the family $\left\{\mathcal{A}_{\lambda}\right\}(\lambda \in \Lambda)$ is equi-attracting.

Proof. Assume that $\mathcal{A}_{\lambda}$ is continuous in $\lambda$ with respect to the Hausdorff distance. Let $B$ be a bounded subset of $X$, and $\varepsilon>0$. We need to prove that there exists a $\tau=\tau(B, \varepsilon) \in \mathbb{T}$ independent of $\lambda \in \Lambda$ such that

$$
d_{H}\left(S_{\lambda}(t) B, \mathcal{A}_{\lambda}\right)<\varepsilon, \quad \forall t \geq \tau, \forall \lambda \in \Lambda,
$$

First, by the compactness of $\Lambda$, it is easy to check that $\mathcal{A}_{\lambda}$ is uniformly continuous in $\lambda$ with respect to the Hausdorff distance. Thus for $r$ sufficiently small, we have

$$
\delta_{H}\left(\mathcal{A}_{\lambda^{\prime}}, \mathcal{A}_{\lambda}\right)<\varepsilon / 2, \quad \forall \lambda, \lambda^{\prime} \in \Lambda, \rho\left(\lambda^{\prime}, \lambda\right) \leq r,
$$

which follows that

$$
\delta_{H}\left(\mathcal{A}_{\lambda}[r], \mathcal{A}_{\lambda}\right) \leq \varepsilon / 2, \quad \forall \lambda \in \Lambda
$$

Now we fix a $r>0$ so that (2.9) holds and consider $\mathcal{A}_{\lambda}[r]$. It follows from Theorem 2.3 that $\left\{\mathcal{A}_{\lambda}[r]\right\}$ equi-attracts $B$. So there exists a $\tau:=\tau(B, \varepsilon) \in \mathbb{T}$ independent of $\lambda$ such that

$$
d_{H}\left(S_{\lambda}(t) B, \mathcal{A}_{\lambda}[r]\right)<\varepsilon / 2, \quad \forall t \geq \tau, \quad \forall \lambda \in \Lambda .
$$

Combining this and (2.9), one concludes that for $t \geq \tau$,

$$
d_{H}\left(S_{\lambda}(t) x, \mathcal{A}_{\lambda}\right) \leq d_{H}\left(S_{\lambda}(t) x, \mathcal{A}_{\lambda}[r]\right)+d_{H}\left(\mathcal{A}_{\lambda}[r], \mathcal{A}_{\lambda}\right)<\varepsilon, \quad \forall \lambda \in \Lambda .
$$

This completes the proof.

REMARK 2.5. Attraction properties like that considered here for dynamical systems under perturbations or discretizations have already attracted notable attention in recent years; see Grüne [4, 5], Kostin and Pilyugin [14] and Stuart and Humphries [20] etc. In particular, in [14], the authors considered a family of discrete dynamical systems generated by mappings $\Sigma_{\lambda}$ in a Hilbert space $H$. They have provided conditions to ensure a $\lambda$-independent exponential attraction rate for the family $\left\{\mathcal{A}_{\lambda}\right\}$ of the global attractors, and thus established certain results which are rather stronger than that of ours in some circumstances.

REMARK 2.6. For gradient systems, it is well known that if the equilibria are hyperbolic, then the global attractors are the union of the equilibria and their unstable manifolds; see [7, 21] etc. This enables us to check the continuous dependence of 
attractors on parameters for many systems arising in applications. For instance, it is proved in [3] that the global attractor $\mathcal{A}_{\lambda}$ of the viscous Cahn-Hilliard equation

$$
(1-\lambda) u_{t}-\lambda \Delta u_{t}+\Delta^{2} u-\Delta f(u)=0
$$

on a bounded domain $\Omega$ equipped with initial and homogeneous Dirichlet boundary conditions, where $\Delta$ is the Laplace operator, is continuous in $\lambda$ as $\lambda$ varies in $[0,1]$. In such situations, one might be able to apply the results obtained here to understand some aspects of the dynamics which are uniform (robust) respect to parameters for the systems under considerations, as we will see in Theorem 2.7 below.

Now we state and prove the uniform Lyapunov stability result for the attractors $\mathcal{A}_{\lambda}$, which is a simple consequence of Theorem 2.4. For this purpose, we need to impose on $S_{\lambda}(t)$ the following uniform continuity assumption.

(C2) For any bounded subset $B$ of $X$ and $T>0, S_{\lambda}(t) x$ is uniformly continuous in $x$ on $B$ in a uniform manner with respect to $\lambda \in \Lambda$ and $t \leq T$, i.e., for any $\varepsilon>0$, there exists a $\delta>0$ such that for all $x, y \in B$ with $d(x, y)<\delta$,

$$
d\left(S_{\lambda}(t) x, S_{\lambda}(t) y\right)<\varepsilon, \quad \forall t \leq T, \forall \lambda \in \Lambda .
$$

THEOREM 2.7. In addition to the hypotheses in Theorem 2.3, assume that $S_{\lambda}(t)$ satisfies the uniform continuity assumption (C2). Let $\mathcal{A}_{\lambda}$ be the global attractor of $S_{\lambda}(t)$. If $\mathcal{A}_{\lambda}$ is continuous in $\lambda$, then the family $\left\{\mathcal{A}_{\lambda}\right\}$ is uniformly Lyapunov stable; i.e., for any $\varepsilon>0$, there exists a $\delta>0$ independent of $\lambda$ such that for all $\lambda \in \Lambda$,

$$
d\left(S_{\lambda}(t) x, \mathcal{A}_{\lambda}\right)<\varepsilon, \quad \forall t \in \mathbb{T},
$$

provided $d\left(x, \mathcal{A}_{\lambda}\right)<\delta$.

REMARK 2.8. The converse of the conclusion in Theorem 2.7 fails to be true; i.e., uniform Lyapunov stability of $\left\{\mathcal{A}_{\lambda}\right\}$ does not imply continuity of $\mathcal{A}_{\lambda}$ in $\lambda$ in the general case; see Remark 3.3.

Proof of Theorem 2.7. Let $\mathcal{U}$ be the bounded subset of $X$ appearing in (2.2). By (2.2), it is easy to see that $\mathcal{A}_{\lambda} \subset \mathcal{U}$ for all $\lambda \in \Lambda$. Set

$$
B=\{x \in X \mid d(x, \mathcal{U}) \leq 1\} .
$$

Since $\mathcal{A}_{\lambda}$ is continuous in $\lambda$, thanks to Theorem 2.4 , we know that $\mathcal{A}_{\lambda}$ equi-attracts $B$ for $\lambda \in \Lambda$.

Let $\varepsilon>0$ be given arbitrary. Then there exists a $T \in \mathbb{T}$ independent of $\lambda$ such that

$$
d_{H}\left(S_{\lambda}(t) B, \mathcal{A}_{\lambda}\right)<\varepsilon, \quad \forall t>T, \forall \lambda \in \Lambda .
$$

On the other hand, by the continuity assumption $(C 2)$ one can find a positive number $\delta<1$ such that for $x, y \in B$ with $d(x, y)<\delta$,

$$
d\left(S_{\lambda}(t) x, S_{\lambda}(t) y\right)<\varepsilon, \quad \forall t \leq T, \forall \lambda \in \Lambda .
$$

Now for any $\lambda \in \Lambda$, we claim that (2.10) holds for all $x \in X$ with $d\left(x, \mathcal{A}_{\lambda}\right)<\delta$. Indeed, if $d\left(x, \mathcal{A}_{\lambda}\right)<\delta$, we can pick a $y \in \mathcal{A}_{\lambda}$ such that $d(x, y)<\delta$. Therefore for $t \leq T$, we 
conclude by (2.12) and $S_{\lambda}(t) y \in \mathcal{A}_{\lambda}$ that

$$
d\left(S_{\lambda}(t) x, \mathcal{A}_{\lambda}\right) \leq d\left(S_{\lambda}(t) x, S_{\lambda}(t) y\right)<\varepsilon .
$$

For $t>T$, note that $d\left(x, \mathcal{A}_{\lambda}\right)<\delta$ implies $x \in B$, the conclusion follows immediately from (2.11).

Finally, let us state and prove the following equivalence result regarding equiattraction and continuous dependence of attractors on parameters.

THEOREM 2.9. In addition to the hypotheses in Theorem 2.3, assume that $S_{\lambda}(t) x$ is equi-continuous in $\lambda$ for $(t, x)$ in any bounded subset of $\mathbb{T} \times X$. Let $\mathcal{A}_{\lambda}$ be the global attractor of $S_{\lambda}(t)$. Then $\left\{\mathcal{A}_{\lambda}\right\}$ is equi-attracting iff $\mathcal{A}_{\lambda}$ is continuous in $\lambda$ with respect to the Hausdorff distance.

Proof. We only need to prove the "only if" part. So we assume that $\left\{\mathcal{A}_{\lambda}\right\}$ equiattracts each bounded subset of $X$.

Let $\mathcal{U}$ be the bounded subset of $X$ in (2.2). Clearly $\mathcal{A}_{\lambda} \subset \mathcal{U}$ for all $\lambda \in \Lambda$. Let $\varepsilon>0$. By the equi-attraction of $\left\{\mathcal{A}_{\lambda}\right\}$, there exists a $\tau \in \mathbb{T}$ independent of $\lambda$ such that, for any $\lambda \in \Lambda, d_{H}\left(S_{\lambda}(t) \mathcal{U}, \mathcal{A}_{\lambda}\right)<\varepsilon$ provided $t \geq \tau$. It follows that

$$
d_{H}\left(S_{\lambda}(t) \mathcal{A}_{\lambda^{\prime}}, \mathcal{A}_{\lambda}\right)<\varepsilon, \quad \forall t \geq \tau, \quad \forall \lambda, \lambda^{\prime} \in \Lambda .
$$

By the equi-continuity assumption on $S_{\lambda}(t) x$ in $\lambda$, there exists a $\delta>0$ such that

$$
\sup _{x \in \mathcal{U}} d\left(S_{\lambda^{\prime}}(\tau) x, S_{\lambda}(\tau) x\right)<\varepsilon, \quad \forall \lambda, \lambda^{\prime} \in \Lambda \text { with } \rho\left(\lambda^{\prime}, \lambda\right)<\delta .
$$

As a consequence,

$$
d_{H}\left(S_{\lambda^{\prime}}(t) \mathcal{A}_{\lambda^{\prime}}, S_{\lambda}(\tau) \mathcal{A}_{\lambda^{\prime}}\right)<\varepsilon, \quad \forall \lambda, \lambda^{\prime} \in \Lambda \text { with } \rho\left(\lambda^{\prime}, \lambda\right)<\delta,
$$

and hence

$$
\begin{aligned}
d_{H}\left(\mathcal{A}_{\lambda^{\prime}}, \mathcal{A}_{\lambda}\right) & =d_{H}\left(S_{\lambda^{\prime}}(\tau) \mathcal{A}_{\lambda^{\prime}}, \mathcal{A}_{\lambda}\right) \\
& \leq d_{H}\left(S_{\lambda^{\prime}}(\tau) \mathcal{A}_{\lambda^{\prime}}, S_{\lambda}(\tau) \mathcal{A}_{\lambda^{\prime}}\right)+d_{H}\left(S_{\lambda}(\tau) \mathcal{A}_{\lambda^{\prime}}, \mathcal{A}_{\lambda}\right)<2 \varepsilon
\end{aligned}
$$

for any $\lambda, \lambda^{\prime} \in \Lambda$ with $\rho\left(\lambda^{\prime}, \lambda\right)<\delta$, which gives

$$
\delta_{H}\left(\mathcal{A}_{\lambda^{\prime}}, \mathcal{A}_{\lambda}\right)=\max \left(d_{H}\left(\mathcal{A}_{\lambda^{\prime}}, \mathcal{A}_{\lambda}\right), d_{H}\left(\mathcal{A}_{\lambda}, \mathcal{A}_{\lambda^{\prime}}\right)\right)<2 \varepsilon .
$$

This finishes the proof of the desired result.

REMARK 2.10. Although the continuity assumptions on $S_{\lambda}(t)$ in Theorems $2.3-2.5$ seem to be rather strong, they can be verified under quite natural conditions for specific systems. We will illustrate this in Section 3 with an example involving an ordinary differential equation with a parameter $\lambda$.

3. Example. We consider the following ordinary differential system with parameter $\lambda \in \Lambda$

$$
x^{\prime}(t)=f(x(t), \lambda), \quad t \geq 0,
$$


where $\Lambda$ is a compact metric space with metric $\rho$ and the vector field $f: \mathbb{R}^{n} \times \Lambda \rightarrow \mathbb{R}^{n}$ is always assumed to be locally Lipschitz in the space variable $x$. For any $x \in R^{n}$, denote by $\phi_{\lambda}(t ; x)$ the unique solution $x(t)$ of $(3.1)_{\lambda}$ with initial value $x(0)=x$. We assume that

AsSumption (SA). Each solution of $(3.1)_{\lambda}$ exists on $\mathbb{R}^{+} ;$moreover, for any bounded subset $B$ of $\mathbb{R}^{n}$ and $T>0$, there is a constant $C_{T}>0$ independent of $\lambda$ such that

$$
\left|\phi_{\lambda}(t ; x)\right| \leq C_{T}, \quad \forall t \in[0, T], x \in B, \lambda \in \Lambda .
$$

This enables us to define a family of dynamical systems $S_{\lambda}(t)$ on $\mathbb{R}^{n}$ for $\lambda \in \Lambda$,

$$
S_{\lambda}(t) x=\phi_{\lambda}(t ; x), \quad \forall t \geq 0, x \in \mathbb{R}^{n} .
$$

We claim that if $f$ is continuous in $(x, \lambda)$ on $\mathbb{R}^{n} \times \Lambda$, then the continuity assumptions in the theorems of Section 2 are satisfied by $S_{\lambda}(t)$.

Here we only verify that $S_{\lambda}(t) x$ satisfies the equi-continuity assumption in $\lambda$ since the proof for the other ones is easy or follows a similar manner and is thus omitted. Let $B$ be a bounded subset of $\mathbb{R}^{n}$ and $T>0$. We may assume that $B$ is closed. If $S_{\lambda}(t) x$ is not equi-continuous in $\lambda$ with respect to $(t, x) \in[0, T] \times B$, there would exist sequences $\lambda_{n}, \lambda_{n}^{\prime} \in \Lambda$ with $\rho\left(\lambda_{n}, \lambda_{n}^{\prime}\right) \rightarrow 0$ and $\left(t_{n}, x_{n}\right) \in[0, T] \times B$ such that for some $\varepsilon_{0}>0$,

$$
\left|S_{\lambda_{n}}\left(t_{n}\right) x_{n}-S_{\lambda_{n}^{\prime}}\left(t_{n}\right) x_{n}\right| \geq \varepsilon_{0}, \quad n \in N .
$$

Due to the compactness, it can be assumed that $\lambda_{n}, \lambda_{n}^{\prime} \rightarrow \lambda_{0}$ and $\left(t_{n}, x_{n}\right) \rightarrow\left(t_{0}, x_{0}\right)$. Let $x_{n}(t)=S_{\lambda_{n}}(t) x_{n}, y_{n}(t)=S_{\lambda_{n}^{\prime}}(t) x_{n}$. Then

$$
\begin{gathered}
x_{n}^{\prime}(t)=f\left(x_{n}(t), \lambda_{n}\right), \quad y_{n}^{\prime}(t)=f\left(y_{n}(t), \lambda_{n}^{\prime}\right), \\
x_{n}(0)=y_{n}(0)=x_{n} .
\end{gathered}
$$

By Assumption (SA), we see that $x_{n}(t)$ and $y_{n}(t)$ are uniformly bounded on $[0, T]$. It follows by (3.5) and the joint continuity of $f(x, \lambda)$ in $(x, \lambda)$ that $x_{n}^{\prime}(t)$ and $y_{n}^{\prime}(t)$ are uniformly bounded on $[0, T]$. By the Arzela-Ascoli Theorem, there exists a subsequence $n_{k}$ of $n$ such that $x_{n_{k}}(t)$ and $y_{n_{k}}(t)$ converge uniformly on $[0, T]$ to functions $x_{0}(t)$ and $y_{0}(t)$, respectively. Using (3.5) and the joint continuity of $f(x, \lambda)$ in $(x, \lambda)$ once again, one sees that $x_{n_{k}}^{\prime}(t)$ and $y_{n_{k}}^{\prime}(t)$ converge uniformly on $[0, T]$. Hence we necessarily have $x_{0}(t), y_{0}(t) \in C^{1}\left([0, T] ; \mathbb{R}^{n}\right)$; moreover, $x_{n_{k}}(t)$ and $y_{n_{k}}(t)$ converge in $C^{1}\left([0, T] ; \mathbb{R}^{n}\right)$ to $x_{0}(t)$ and $y_{0}(t)$, respectively. Passing to the limit in (3.5) and (3.6), we find that $x_{0}(t)$ and $y_{0}(t)$ are both solutions of $(3.1)_{\lambda_{0}}$ on $[0, T]$ with initial value $x_{0}$. Therefore $x_{0}(t) \equiv y_{0}(t)$ on $[0, T]$. Thus it follows that for any $\varepsilon>0$, $\max _{0 \leq t \leq T}\left|x_{n_{k}}(t)-y_{n_{k}}(t)\right|<\varepsilon$ when $k$ is sufficiently large. However, this contradicts (3.4).

The following is a direct application of the theorems in Section 2.

THeOREM 3.1. Assume that $f \in C\left(\mathbb{R}^{n} \times \Lambda\right)$ and satisfies Assumption $(S A)$. Suppose that the family of the solution semigroup $\left\{S_{\lambda}(t)\right\}$ of $(3.1)_{\lambda}$ is equi-dissipative on $\mathbb{R}^{n}$. Let $\mathcal{A}_{\lambda}$ be the global attractor of $S_{\lambda}(t)$. Then

(1) For any $r>0$ fixed, the family $\left\{\mathcal{A}_{\lambda}[r]\right\}$ is equi-attracting, where $\mathcal{A}_{\lambda}[r]$ is as in (1.2).

(2) $\left\{\mathcal{A}_{\lambda}\right\}$ is equi-attracting iff $\mathcal{A}_{\lambda}$ is continuous in $\lambda$ with respect to the Hausdorff distance. 


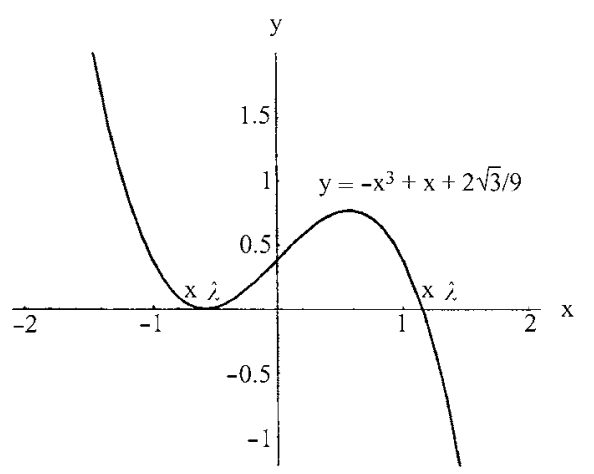

Fig. 1. Two equilibria at $\lambda_{0}$

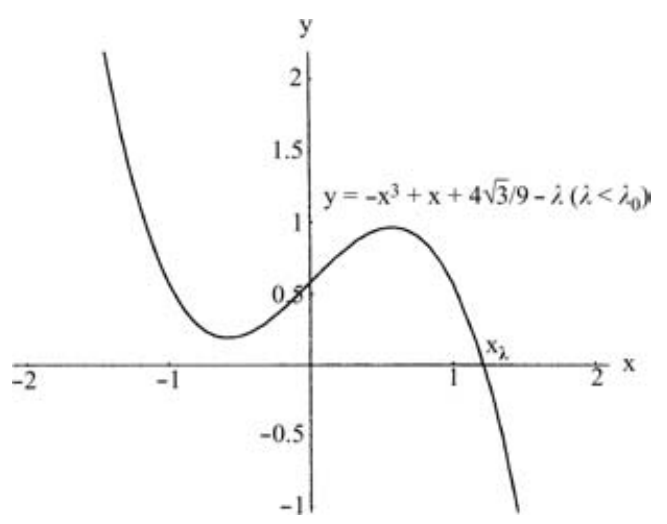

Fig. 2. One equilibrium for $\lambda<\lambda_{0}$

The following example shows that a singularity (with respect to parameters) may appear in the rate of attraction of the global attractors even in quite simple cases.

EXAMPLE 3.2. For $\lambda \in\left[0, \lambda_{0}\right]$, where $\lambda_{0}=2 \sqrt{3} / 9$, consider the scalar equation

$$
x^{\prime}(t)=-x^{3}(t)+x(t)+4 \sqrt{3} / 9-\lambda .
$$

For $\lambda=\lambda_{0}$, the equation has two distinct equilibria $x_{\lambda_{0}}^{-}$and $x_{\lambda_{0}}^{+}$(see Fig. 1), where

$$
x_{\lambda_{0}}^{-}=-\sqrt{3} / 3, \quad x_{\lambda_{0}}^{+}>0
$$

and for $\lambda<\lambda_{0}$, it has only one equilibrium $x_{\lambda}$, which is continuous in $\lambda$ (see Fig. 2).

Let $S_{\lambda}(t)$ be the solution semigroup of $(3.7)_{\lambda}$. For Eq. $(3.7)_{\lambda}$, it is easy to check that all the conditions in Theorem 3.1 are fulfilled.

Let $\lambda<\lambda_{0}$. We observe (see Fig. 2) that

$$
-x^{3}+x+4 \sqrt{3} / 9-\lambda>0, \quad \text { for } x<x_{\lambda}
$$

and

$$
-x^{3}+x+4 \sqrt{3} / 9-\lambda<0, \quad \text { for } x>x_{\lambda},
$$


from which and the equation (3.7) $\lambda$ it can be easily seen that $S_{\lambda}(t) x \rightarrow x_{\lambda}$ as $t \rightarrow+\infty$ for any $x \in \mathbb{R}^{1}$; moreover, for any $\varepsilon>0,\left(x_{\lambda}-\varepsilon, x_{\lambda}+\varepsilon\right)$ is positively invariant under $S_{\lambda}(t)$. Thus $x_{\lambda}$ is globally asymptotically stable. Thanks to the basic theory of dynamical systems (see $[\mathbf{1}, \mathbf{7}, \mathbf{1 7}]$ etc.), we know that $x_{\lambda}$ attracts each bounded subset of $\mathbb{R}^{1}$. Hence the global attractor $\mathcal{A}_{\lambda}$ is precisely the equilibrium $x_{\lambda}$.

For $\lambda=\lambda_{0}$, the global attractor $\mathcal{A}_{\lambda_{0}}$ of $S_{\lambda_{0}}(t)$ contains at least two points $x_{\lambda_{0}}^{-}$ and $x_{\lambda_{0}}^{+}$. By Lemma 1.3 in [21] Chap. 1, we know that $\mathcal{A}_{\lambda_{0}}$ is connected, and hence $\left[x_{\lambda_{0}}^{-}, x_{\lambda_{0}}^{+}\right] \subset \mathcal{A}_{\lambda_{0}}$. One also observes that $S_{\lambda_{0}}(t) x \rightarrow x_{\lambda_{0}}^{-}$for $x<x_{\lambda_{0}}^{-}$and $S_{\lambda_{0}}(t) x \rightarrow x_{\lambda_{0}}^{+}$ for $x>x_{\lambda_{0}}^{+}$as $t \rightarrow+\infty$. Therefore we conclude that $\mathcal{A}_{\lambda_{0}}=\left[x_{\lambda_{0}}^{-}, x_{\lambda_{0}}^{+}\right]$. It is also easy to check that for any $\varepsilon>0,\left(x_{\lambda_{0}}^{-}-\varepsilon, x_{\lambda_{0}}^{+}+\varepsilon\right)$ is positively invariant under $S_{\lambda_{0}}(t)$.

By virtue of Theorem 3.1, for any $0<\delta<\lambda_{0}$, the family $\left\{\mathcal{A}_{\lambda}\right\}$ is equi-attracting for $\lambda \in[0, \delta]$. However, since $\mathcal{A}_{\lambda}$ is not continuous in $\lambda$ at $\lambda=\lambda_{0}$ with respect to the Hausdorff distance, it does not possess equi-attraction property for $\lambda \in\left[0, \lambda_{0}\right]$. For $\varepsilon>0$ and bounded subset $B$ of $\mathbb{R}^{1}$, denote by $\tau_{\lambda}(B, \varepsilon)$ the minimal time such that

$$
d_{H}\left(S_{\lambda}(t) B, \mathcal{A}_{\lambda}\right)<\varepsilon, \quad \text { for } t>\tau_{\lambda}(B, \varepsilon) .
$$

Then there exist a $\varepsilon_{0}>0$, a bounded subset $B_{0}$ of $\mathbb{R}^{1}$, and a sequence $\lambda_{n} \rightarrow \lambda_{0}$ such that

$$
\tau_{\lambda_{n}}\left(B_{0}, \varepsilon_{0}\right) \rightarrow+\infty \quad \text { as } \lambda_{n} \rightarrow \lambda_{0}
$$

Hence, there is a singularity in the attraction rate of $\mathcal{A}_{\lambda}$ as $\lambda \rightarrow \lambda_{0}$.

On the other hand, Theorem 3.1 also tells us that for any $r>0$ fixed, the family of parameterically inflated attractors $\left\{\mathcal{A}_{\lambda}[r]\right\}$ equi-attracts each bounded subset $B$ of $\mathbb{R}^{1}$ for $\lambda \in\left[0, \lambda_{0}\right]$.

REMARK 3.3. Since for any $\varepsilon>0,\left(x_{\lambda}-\varepsilon, x_{\lambda}+\varepsilon\right)\left(\lambda<\lambda_{0}\right)$ and $\left(x_{\lambda_{0}}^{-}-\varepsilon, x_{\lambda_{0}}^{+}+\varepsilon\right)$ are positively invariant under $S_{\lambda}(t)$ and $S_{\lambda_{0}}(t)$, respectively, we see that the family of attractors $\left\{\mathcal{A}_{\lambda}\right\}$ is uniformly Lyapunov stable for $\lambda \in\left[0, \lambda_{0}\right]$. However, $\mathcal{A}_{\lambda}$ is not continuous at $\lambda=\lambda_{0}$. This shows that the converse of the conclusion in Theorem 2.7 may fail to be true in the general case.

ACKNOWLEDGMENT. The authors would like to express their gratitude to the referee for his valuable comments and suggestions which greatly improved the quality of the paper.

\section{REFERENCES}

1. F. H. Clarke, Yu. S. Ledyaev and R. J. Stern, Asymptotic stability and smooth Lyapunov functions, J. Diff. Eqns. 149 (1998), 69-114.

2. J. W. Cholewa and T. Dlotko, Global attractors in abstract parabolic problems, London Math. Soc. Lecture Note Series, No. 278 (Cambridge University Press, 2000).

3. C. M. Elliott and A. M. Stuart, Viscous Cahn-Hilliard equation, J. Diff. Equations 128 (1996), 387-414.

4. L. Grüne, Convergence rates of perturbed attracting sets with vanishing perturbations, J. Math. Anal. Appl. 244 (2000), 369-392.

5. L. Grüne, Asymptotic behavior of dynamical and control systems under perturbation and discretization, Lecture Notes in Mathematics No. 1783 (Springer-Verlag, 2002).

6. Boling Guo, Nonlinear evolution equations (Shanghai Scientific and Technological Education Publishing House, Shanghai, 1995), (in Chinese). 

1988).

7. J. K. Hale, Asymptotic behavior of dissipative systems (Amer. Math. Soc., Providence,

8. J. K. Hale and G. Raugel, Lower semicontinuity of attractors of gradient systens and applications, Ann. Math. Pura Appl. 154 (1989), 281-326.

9. J. K. Hale, X. B. Lin and G. Raugel, Upper semicontinuity of attractors for approximations of semigroups and partial differential equations, Math. Comp. 50 (1988), 89-123.

10. J. K. Hale, Dynamics of numerical approximations, Appl. Math. Comp. 89 (1998), 5-15.

11. A. Haraux, Attractors of asymptotically compact processes and applications to nonlinear partial equations, Comm. Partial Differential Equations 13 (1988), 1383-1414.

12. P. E. Kloeden and V. S. Kozyakin, The inflation of attractors and discretization: the autonomous case, Nonlinear Anal. TMA 40 (2000), 333-343.

13. P. E. Kloeden and V. S. Kozyakin, Uniform nonautonomous attractors under discretization, Discrete Contin. Dyn. Systems 10 (2004), 423-433.

14. I. N. Kostin and S. Yu. Pilyugin, Uniform exponential stability of the attractors of perturbed evolution equations, Dokl. Akad. Nauk 369 (1999), 449-450. (Russian)

15. I. N. Kostin, Lower semicontinuity of a non-hyperbolic attractor, J. London Math. Soc. (2) 52 (1995), 568-582.

16. D. S. Li and X. X. Zhang, Strongly positively invariant attractors for periodic processes, J. Math. Anal. Appl. 241 (2000), 10-29.

17. D. S. Li, On the stability in general dynamical systems and differential inclusions, J. Math. Anal. Appl. 274 (2002), 705-724.

18. J. C. Robinson, Infinite-dimensional dynamical systems (Cambridge University Press, Cambridge 2001).

19. G. P. Sell and Y. C. You, Dynamics of evolutionary equations (Springer-Verlag, 2002).

20. A. M. Stuart and A. R. Humphries, Numerical analysis and dynamical systems (Cambridge University Press, 1996).

21. R. Temam, Infinite dimensional dynamical systems in mechanics and physics (SpringerVerlag, 1988).

22. M. I. Vishik, Asymptotic behaviour of solutions of evolutionary equations (Cambridge University Press, 1992).

23. C. K. Zhong, D. S. Li and P. E. Kloeden, Uniform attractors of periodic and asymptotic periodic dynamical systems. Discrete Contin. Dyn. Systems, to appear. 